\title{
An Investigation into Initial Ranging Method for Mobile OFDMA Systems
}

\author{
Jianqiang Zeng and Hlaing Minn (Contact Author) \\ Department of Electrical Engineering, University of Texas at Dallas \\ Email: $\{$ jxz051000, hlaing.minn $\} @$ utdallas.edu
}

\begin{abstract}
This paper addresses initial ranging, which includes multiuser ranging code detection, multiuser timing offset estimation and multiuser power estimation at the base station for TDD OFDMA systems. By means of ranging signal design, our proposed ranging method overcomes the existing ranging method's drawbacks namely high complexity, high sensitivity to channel frequency selectivity and time selectivity. Comparisons with the existing methods [1] [2] corroborate the advantages of our method in time-varying mobile environment.
\end{abstract}

\section{INTRODUCTION}

Orthogonal frequency division multiple access (OFDMA) has recently drawn much attention and has been adopted as one of the three physical layer modes in the IEEE wireless MAN standards 802.16e [3]. In OFDMA systems, uplink synchronization is accomplished by initial ranging process. In the current initial ranging scheme defined in IEEE 802.16e [3], each ranging subscriber station (RSS) first attains downlink synchronization and uplink transmission parameters from downlink control frames, and then it randomly chooses one of the available ranging codes and transmits it twice over two consecutive symbols on a single ranging channel that consists of 144 non-contiguous sub-carriers specified in the UL-MAP message. After detecting ranging codes and extracting the information of timing and power from the received ranging signal, the base station (BS) will broadcast a ranging response message that advertises the received ranging code and the ranging time-slot where the ranging code has been identified. The ranging response message also contains all the adjustment information (e.g., timing and power adjustment) and a status notification (e.g., success, continue). If an RSS receives the success notification, its ranging process is complete. Otherwise the RSS continues the ranging process until it receives the success notification.

The ranging methods proposed in [1] and [4] both use a frequency domain correlator bank to detect the ranging codes and estimate the timing offsets. The method in [1] first pre-computes phase-compensated ranging codes to account for the effects of timing offsets. For a timing offset range of $\left[0, d_{\max }\right]$ samples, each of the $N_{c}$ ranging codes gives $\left(d_{\max }+1\right)$ phase-compensated codes. Each of these $N_{c}\left(d_{\max }+1\right)$ phase-compensated codes is cross-correlated with the received frequency-domain symbols on the ranging channel in every ranging time slot to detect the codes and estimate the timing offsets. The method in [4] applies the approach in [1] only to the ranging time slots where the presence of ranging codes have been identified by an energy detector. The ranging method in [5] uses a similar crosscorrelation approach but in the time domain. Common disadvantages of these existing ranging methods are the high computational complexity and inherent vulnerability to the channel frequency-selectivity and time-selectivity and other users' interferences. By means of a ranging signal design, the ranging method in [2] achieves low-complexity ranging signal detection. It does not use frequency domain CDMA codes of the methods in [1], [4], [5] but utilizes the orthogonality principle and the best channel identification conditions. This method assumes that the channel remains constant over $M_{r}$ OFDM symbol intervals, which is a reasonable assumption for fixed wireless communication systems such as IEEE 802.162004 [6], but it is not true for mobile communication at vehicular speeds. When the channels vary during $M_{r}$ OFDM symbol intervals, the orthogonality is destroyed. This will introduce inter-carrier interference (ICI) and hence degrade the ranging performance.

In this paper, we present a new ranging signal design for mobile OFDMA systems which overcomes the existing method's drawbacks. The proposed ranging signal design divides the different ranging signals into several groups. The signals from different groups are transmitted on different subcarriers. The ranging signals in the same group are transmitted on different ranging time slots instead of being transmitted over $M_{r}$ symbol intervals in [2]. The ICI effect can be reduced by our ranging signal design and hence a better ranging performance can be achieved.

The rest of this paper is organized as follows. The system and signal models are described in Section II. The proposed ranging method is presented in Section III. Simulation results and discussion are given in Section IV, and conclusions are drawn in Section V.

\section{System Description And Signal Model}

Consider an uplink OFDMA system with $N_{r}$ RSSs, $N_{d}$ data subscriber stations (DSSs) and $N$ sub-carriers. After excluding DC and null sub-carriers, the remaining sub-carriers are grouped into $Q_{r}$ ranging sub-channels and $Q_{d}$ data subchannels. The numbers of left and right null sub-carriers are denoted by $\gamma_{L N}$ and $\gamma_{R N}$, respectively. Each ranging subchannel has $\gamma_{r}$ sub-carriers and each data sub-channel has $\gamma_{d}$ sub-carriers. The indices of the subcarriers corresponding to the $q$-th ranging subchannel $\left(q \in\left\{0, \ldots, Q_{r}-1\right\}\right)$ and the 
$q_{d}$-th data subchannel $\left(q_{d} \in\left\{0, \ldots, Q_{d}-1\right\}\right)$ are denoted as $\mathcal{J}_{q}$ and $\mathcal{K}_{q_{d}}$, respectively. One UL frame consists of $M$ OFDM symbols where predefined subcarriers over $M_{r}$ OFDM symbols are allocated for the ranging. The ranging time slot index is denoted by $t\left(t \in\left\{0,1, \ldots, \frac{M_{r}}{M_{1}}-1\right\}\right)$, where $M_{1}$ is the number of OFDM symbols in one ranging time slot. The indices of OFDM symbols in one ranging time slot are denoted by $m_{1}\left(m_{1} \in\left\{0,1, \ldots, M_{1}-1\right\}\right)$.

In the subcarrier domain at the $m$-th OFDM symbol interval, the length- $N$ ranging code vector for the $i$-th RSS and the length- $N$ data vector for the $j$-th DSS are denoted by $\mathbf{X}_{i, r}^{(m)}$ and $\mathbf{X}_{j, d}^{(m)}$, respectively. The corresponding $k$-th elements $(k \in$ $\{0, \ldots, N-1\})$ are given by

$\mathrm{X}_{i, r}^{(m)}(k)= \begin{cases}A_{i, r} C_{i, r}^{\left(m_{1}\right)}(l), & k \in \mathcal{J}_{q}(l), l=0, \ldots, \gamma_{r}-1, \\ & m=M_{1} \cdot t+m_{1} \\ 0, & \text { otherwise }\end{cases}$

$\mathrm{X}_{j, d}^{(m)}(k)= \begin{cases}A_{j, d} C_{j, d}^{(m)}(l), & k \in \mathcal{K}_{q_{d}}(l), l=0, \ldots, \gamma_{d}-1, \\ & m=0, \ldots, M-1 \\ 0, & \text { otherwise }\end{cases}$

where $C_{i, r}\left(m_{1}\right)(l)$ and $C_{j, d}^{(m)}(l)$ are ranging and data symbols, where $C_{i, r}^{\left(m_{1}\right)}(l)$ and $C_{j, d}^{(m)}(l)$ are ranging and data symbols, respectively, with $\left|C_{i, r}^{\left(m_{1}\right)}(l)\right|=E\left[\left|C_{j, d}^{(m)}(l)\right|^{2}\right]=1$, and $\left\{A_{i, r}, A_{j, d}>0\right\}$ are amplitude scaling factors. Denote the unitary $N$-point inverse discrete Fourier transform $\left(\mathrm{IDFT}_{N}\right)$ of $\mathbf{X}_{i, r}^{(m)}$ and $\mathbf{X}_{j, d}^{(m)}$ by $\left[\mathrm{x}_{i, r}^{(m)}(0), \ldots, \mathbf{x}_{i, r}^{(m)}(N-1)\right]^{T}$ and $\left[\mathrm{x}_{i, d}^{(m)}(0), \ldots, \mathrm{x}_{i, d}^{(m)}(N-1)\right]^{T}$. The last $N_{g}$ samples of the IDFT $_{N}$ output are copied and inserted as the cyclic prefix (CP) to avoid inter-symbol interference (ISI), i.e., $\mathrm{x}_{i, \star}^{(m)}(-l)=$ $\mathrm{x}_{i, \star}^{(m)}(N-l)$ for $l=1,2, \ldots, N_{g} \cdot{ }^{1}$ So the time domain signal samples of the $i$-th RSS or DSS are given by

$\mathrm{x}_{i, \star}(n)= \begin{cases}\mathrm{x}_{i, \star}^{(m)}\left(l-N_{g}\right), & n=m\left(N+N_{g}\right)+l \\ & l=0, \ldots, N+N_{g}-1 \\ & m=0, \ldots, M_{r}-1 \text { (for RSS) } \\ & m=0, \ldots, M-1 \text { (for DSS) } \\ & \text { otherwise. }\end{cases}$

We consider independent multi-path Rayleigh fading channels. The sample-spaced channel impulse response taps (including the transmit and receive filters) for the $i$-th RSS/DSS are denoted by $\left\{h_{i, \star}(l), l=0, \ldots, L-1\right\}$. The channel output samples for the $i$-th RSS/DSS are given by

$$
y_{i, \star}(n)=\sum_{l=0}^{L-1} h_{i, \star}(l) x_{i, \star}\left(n-l-d_{i, \star}\right)
$$

where $d_{i, r}\left(d_{i, d}\right)$ is the transmission delay for the $i$-th RSS (DSS). Then the time domain received signal at the BS can be expressed as

$$
y(n)=\sum_{i=0}^{N_{r}-1} y_{i, r}(n)+\sum_{j=0}^{N_{d}-1} y_{j, d}(n)+\omega(n)
$$

\footnotetext{
${ }^{1}$ In the rest of the paper, the subscript $\star$ denotes whether $r$ or $d$.
}

where $\{\omega(n)\}$ are independent and identically-distributed (i.i.d.), circularly-symmetric complex Gaussian noise samples with zero mean and variance $\sigma_{\omega}^{2}=E\left[|\omega(n)|^{2}\right]$.

\section{PROPOSED RANGING METHOD}

Consider an interleaved OFDMA system with $Q_{r}$ ranging sub-channels, where each ranging sub-channel is composed of $\gamma_{r}$ sub-carriers which are spread out over the whole frequency band with a cyclically equal spacing of $\frac{N}{\gamma_{r}}$ sub-carriers. Each RSS uses $\gamma_{r}$ sub-carriers (i.e., one ranging sub-channel) and one OFDM symbol. So the total number of ranging opportunities (distinct ranging signals) is $N_{c}=Q_{r} M_{r}$.

We assume that there are no collisions, that means the different RSSs will pick the different ranging opportunities. The $N_{c}$ different ranging signals are divided into $Q_{r}$ groups. The ranging signals from different groups are transmitted on different sub-carriers. The sub-carrier assignment for the $q$-th ranging subchannel is defined by

$$
\mathcal{J}_{q}=\left\{\left(\frac{l N}{\gamma_{r}}+\Delta_{q_{M_{r}}}\right): l=0, \cdots, \gamma_{r}-1\right\}
$$

where

$$
0 \leq \Delta_{q_{M_{r}}}<\frac{N}{\gamma_{r}} \& \Delta_{q_{M_{r}}} \neq \Delta_{k_{M_{r}}} \text { if } q_{M_{r}} \neq k_{M_{r}} .
$$

$q_{M_{r}}=\left\lfloor q / M_{r}\right\rfloor$ hwere $\lfloor\cdot\rfloor$ denotes floor operation.

The $M_{r}$ ranging signals in the same group will be transmitted on different ranging time slots, and hence can be easily decoupled. While for the ranging method in [2], the $M_{r}$ ranging signals in the same group are overlapped in the time domain, and their decoupling requires processing over $M_{r}$ symbols rendering the loss of the ranging signal orthogonality in the mobile environment.

The proposed method first performs multiuser ranging signal detection based on the BS timing reference. Then utilizing the ranging signal detection results, the received power estimations and timing offsets estimation are performed for each detected RSS.

\section{A. Multiuser Ranging Signal Detection}

At the BS after the CP removal and the $N$-point DFT operation, the frequency domain signal at the $k$-th sub-carrier and the $m$-th symbol interval can be expressed as

$$
\begin{aligned}
Y^{(m)}(k)= & \sum_{i=0}^{N_{r}-1} X_{i, r}^{(m)}(k) H_{i, r}(k)+\sum_{j=0}^{N_{d}-1} X_{j, d}^{(m)}(k) H_{j, d}(k) \\
& +W^{(m)}(k)
\end{aligned}
$$

where

$$
\begin{aligned}
& H_{i, \star}^{(m)}(k)=e^{\frac{j 2 \pi k d_{i, \star}}{N}} \sum_{l=0}^{L-1} h_{i, \star}(l) e^{\frac{-j 2 \pi l k}{N}}=e^{\frac{j 2 \pi k d_{i, \star}}{N}} \widetilde{H}_{i, \star}^{(m)}(k) \\
& W^{(m)}(k)=\frac{1}{\sqrt{N}} \sum_{l=0}^{N-1} \omega\left(m\left(N+N_{g}\right)+N_{g}+l\right) e^{\frac{-j 2 \pi l k}{N}} .
\end{aligned}
$$




$$
D_{q}=\sum_{k \in \mathcal{J}_{q}} Y^{(m)}(k)\left(Y^{(m)}(k)\right)^{*}= \begin{cases}\sum_{k \in \mathcal{J}_{q}}\left\|X_{i, r}^{(m)}(k) H_{i, r}^{(m)}(k)+W^{(m)}(k)\right\|^{2}, & \text { RSS present } \\ \sum_{k \in \mathcal{J}_{q}}\left\|W^{(m)}(k)\right\|^{2}, & \text { RSS absent }\end{cases}
$$

Ranging codes using different ranging sub-channels are already decoupled in the frequency-domain. Within each group of ranging codes using the same ranging sub-channels, they are disjointed for different ranging time slots. So the decision variable $D_{q}$ to decide the presence of the ranging signal on the $q$-th ranging subchannel is given in equation (11).

The ranging signal detector decides that the ranging signal is detected if $D_{q}>\eta$, where $\eta$ is the detection threshold. The decision variable $D_{q}$ is not affected by other RSSs and DSSs, resulting in an efficient, low complexity multiuser ranging signal detection.

\section{B. Power Estimation}

After multiuser ranging signal detection, the next step in the ranging process is to estimate the corresponding (normalized) received power $P_{q}$ for each detected RSS. The $P_{q}$ is defined by

$$
P_{q}=\frac{\sum_{k \in \mathcal{J}_{q}}\left|X_{i, r}^{(m)}(k)\right|^{2}\left|H_{i, r}^{(m)}(k)\right|^{2}}{N}
$$

From (11) and (12), the received power $P_{q}$ can be expressed as in (13). Since the last term in the nominator has a zero mean, the received power of the detected ranging signals can be estimated as

$$
\hat{P}_{q}=\frac{D_{q}-\sigma_{w}^{2} \gamma_{r}}{N} .
$$

\section{Timing Offset Estimation}

The timing offset estimator used here has the same structure as the timing offset estimator proposed in [2]. The timing offset estimator exploits the orthogonal ranging signal design and the signal redundancy introduced by CPs.

It first reconstructs the $\mathrm{CP}$ of ranging signals since the $\mathrm{CP}$ contains correct timing information. The timing offset estimate for the $i$-th RSS can be obtained by maximizing a sliding correlation metric between the clean version and the interference-affected version of $\mathrm{CP}$ as

$$
\hat{\epsilon}_{j}=\arg \max _{0 \leq \epsilon \leq \epsilon_{\max }} \mathcal{R}\left\{\sum_{m=0}^{M-1}\left(\overline{\mathbf{y}}_{i, r, C P}^{(m)}(\epsilon)\right)^{H} \hat{\mathbf{y}}_{i, r, C P}^{(m)}\right\} .
$$

where $\overline{\mathbf{y}}_{i, r, C P}^{(m)}$ is a clean version of the $m$-th symbol's CP, and $\hat{\mathbf{y}}_{i, r, C P}^{(m)}$ is an interference-affected version CP. The detail of $\overline{\mathbf{y}}_{i, r, C P}^{(m)}$ and $\hat{\mathbf{y}}_{i, r, C P}^{(m)}$ are referred to [2].

A much lower complexity timing offset estimator can be developed by using a modified ranging signal where each ranging subcarrier is replaced by two adjacent ranging subcarriers. The timing offset estimation is now based on the phase angle of the sum of the differential (correlation) between adjacent ranging subcarriers.

\section{SIMULATION RESULTS AND DISCUSSION}

\section{A. Simulation Parameters}

For comparison, the ranging code detector and timing estimator in [1] with the ranging signals from the IEEE 802.16e are used as the reference method 1 (Method1). The ranging method in [2] is used as the reference method 2 (Method2). The system parameters, selected from [3], are summarized in Table I. They are the same for the proposed ranging method and the reference ranging methods.

For the proposed method and Method2, the ranging channel is composed of $Q_{r}=16$ ranging sub-channels, and each ranging sub-channel has $\gamma_{r}=8$ sub-carriers. So the total number of ranging opportunities in the proposed method and in the Method2 are both $N_{c}=16 \cdot 4=64$.

For the Method1, each RSS transmits two selected CDMA code twice on the single ranging channel that consists of 144 non-contiguous sub-carriers over four consecutive symbols. The number of ranging codes is 128 . The total number of ranging opportunities is $N_{c}=\frac{128}{2}=64$ which is the same as that in the proposed method and the Method2.

The time varying channels with different normalized Doppler frequency $f_{d} T_{s}\left(T_{s}=\frac{1}{F_{s}}\right.$ is sample time interval) are simulated using the IDFT approach presented in [7]. The ranging performances for the reference methods and the proposed method are evaluated in the time varying channels with $f_{d} T_{s}=0.00003, f_{d} T_{s}=0.00006, f_{d} T_{s}=0.00009$, and $f_{d} T_{s}=0.00012$, respectively. The corresponding mobile speed are $30 \mathrm{miles} / \mathrm{hr}, 60 \mathrm{miles} / \mathrm{hr}, 90 \mathrm{miles} / \mathrm{hr}$, and 120 miles/hr, respectively.

For each time varying channel conditions, we also evaluated the ranging performance of all three methods with different number of RSSs and different number of DSSs to show how the ranging performance will be affected by RSS's interference and DSS's interference.

Fig. 1 shows the probability of correct detection $\left(P_{c d}\right)$ versus the number of RSSs for the conditions of 0 DSS, 15 DSSs, and 30 DSSs in the different time varying channel conditions. The $P_{c d}$ is defined as $E\left[\frac{D_{c}}{N_{r}}\right]$ where $D_{c}$ is the number of correct detection in one uplink ranging frame. We can see that the proposed method and the Method2 can

$$
P_{q}=\frac{D_{q}-\sum_{k \in \mathcal{J}_{q}}\left|W^{(m)}(k)\right|^{2}-\sum_{k \in \mathcal{J}_{q}} 2 \mathcal{R}\left|X_{i, r}^{(m)}(k) H_{i, r}^{(m)}(k) W^{(m)}(k)\right|}{N}
$$


TABLE I

SYSTEM PARAMETERS

\begin{tabular}{||c|c||}
\hline System channel BW $(\mathrm{MHz})$ & 3 \\
\hline Sampling frequency $F_{s}(\mathrm{MHz})$ & 3.36 \\
\hline FFT size $N$ & 2048 \\
\hline CP length $N_{g}$ & 128 \\
\hline Cell radius & $4.5 \mathrm{~km}$ \\
\hline Maximum RSS timing offset $d_{\max , \mathrm{r}}$ & 104 samples \\
\hline Maximum DSS timing offset $d_{\max , \mathrm{d}}$ & 32 samples \\
\hline Sub-carrier spacing & $1.64 \mathrm{kHz}$ \\
\hline Number of OFDM symbols allocated for ranging $M_{r}$ & 4 \\
\hline Modulation & BPSK for RSS \\
\cline { 2 - 2 } & QPSK for DSS \\
\hline Number of data sub-carriers per sub-channel & 48 \\
\hline Number of channel Taps $L$ & 7 \\
\hline
\end{tabular}
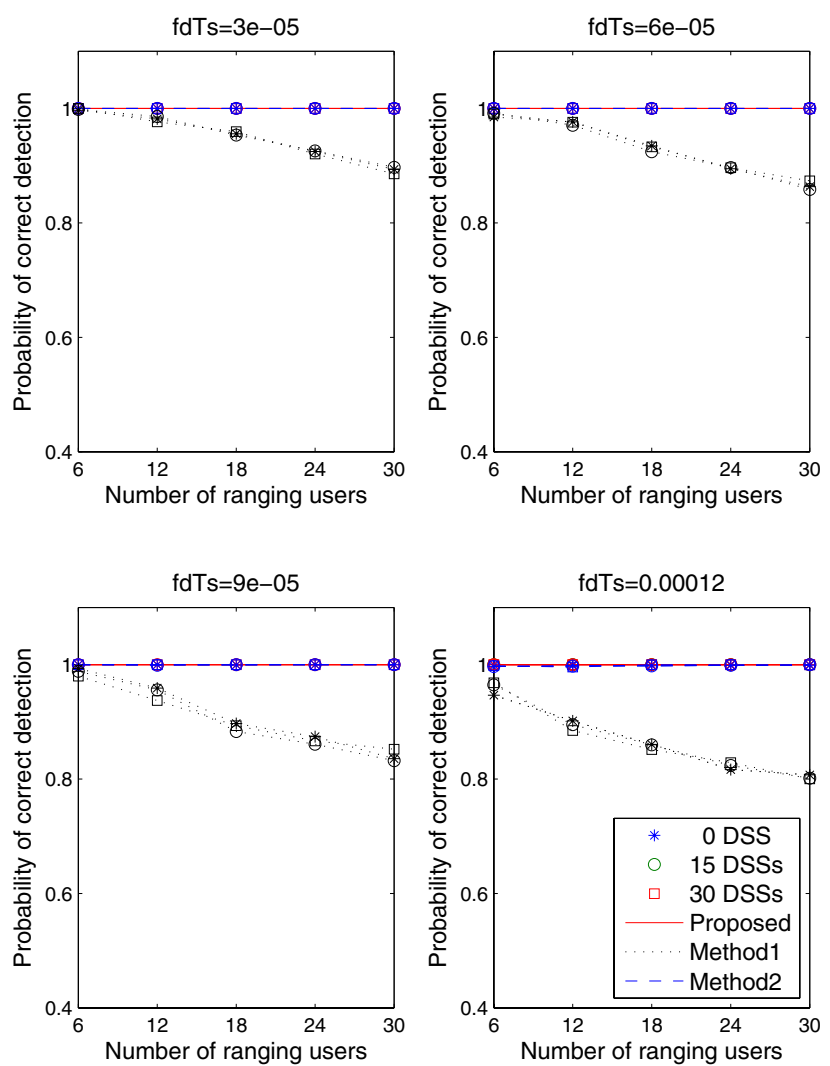

Fig. 1. The probabilities of correct detection

always correctly detect the ranging signals and both are robust to RSS's interference and DSS's interference, while the $P_{c d}$ for the Method1 decreases when the number of the RSSs increases.

Fig. 2 shows the probability of detection false alarm $\left(P_{f a}\right)$ versus the number of RSSs for the conditions of 0 DSS, 15 DSSs, and 30 DSSs in the different time varying channel conditions. The $P_{f a}$ is defined as $E\left[\frac{D_{a}}{N_{c}-N_{r}}\right]$ where $D_{a}$ is the number of ranging signals which are detected at the BS but are not transmitted from any RSSs. The proposed method performs much better than the reference methods. No false alarms are observed for the proposed method, while the reference methods have high $P_{f a}$.

Fig. 3 shows the probability of missed detection $\left(P_{m d}\right)$ versus the number of RSSs for the conditions of 0 DSS, 15 DSSs, and 30 DSSs in the different time varying channel conditions. The $P_{m d}$ is defined as $E\left[\frac{D_{m}}{N_{r}}\right]$ where $D_{m}$ is the number of RSSs whose transmitted ranging signals are not detected at the BS. No missed detections are observed for the proposed method. The Method2 has small amount missed detections observed in the fast varying channel with $f_{d} T_{s}=0.00018$. There are always missed detections observed for the Method1 under both slow and fast varying channel conditions.

Fig. 4 shows the standard deviation of the timing offset estimation versus the number of RSSs for the conditions of 0 DSS, 15 DSSs, and 30 DSSs in the different time varying channel conditions. In each simulation runs, the true timing offsets for RSSs and DSSs are taken randomly from the interval $\left[0, d_{\max , \mathrm{r}}\right]$ and $\left[0, d_{\max , \mathrm{d}}\right]$, respectively. From the figure, we can see that the proposed method has similar performance as the Method2 in the slow varying channel, while in the fast varying environment, the proposed method always performs better than the reference methods.

Fig. 5 shows the normalized power estimation MSE defined as $E\left[\left(1-\frac{\hat{P}}{P}\right)^{2}\right]$ versus the number of RSSs for the conditions of 0 DSS, 15 DSSs, and 30 DSSs in the different time varying channel conditions. Since there is no power estimator provided in Method1, we only compare the performance of the proposed method and the Method2. The proposed method performs much better than the Method2. The performance of the Method2 degrades dramatically when the channel varies fast. The large estimation MSE can be observed in the fast varying channel with $f_{d} T_{s}=0.00018$ due to the ICI caused by channel variations.

\section{CONCLUSIONS}

We investigated and compare several initial ranging methods for OFDMA system in mobile environment. The performance comparisons are presented by simulations. The simulation results show that the proposed method achieves better ranging performance than the existing methods in mobile environment. Hence, the proposed method would be useful in enhancing the IEEE 802.16e standard.

\section{REFERENCES}

[1] J. Krinock, M. Singh, M. Paff, Vincent, A. Lonkar, L. Fung, and C. C. Lee, "Comments on OFDMA ranging scheme described in IEEE 802.16ab-01/01r1," Document IEEE 802.16abc-01/24.

[2] X. Fu, Y. Li, and H. Minn, "A new ranging method for OFDMA systems," IEEE Trans. Wireless Commun., vol. 6, no. 2, pp. 659-669, Feb. 2007.

[3] Broadband Wireless Access: IEEE MAN standard, IEEE LAN/MAN Standards Committee IEEE 802.16e, 2005.

[4] H. Mahmoud, H. Arslan, and M. Ozdemir, "Initial ranging for WiMAX (802.16e) OFDMA," in Proc. IEEE Military Communications Conference, Oct. 2006, pp. 1-7.

[5] X. Fu and H. Minn, "Initial uplink synchronization and power control (ranging process) for OFDMA systems," in Proc. IEEE GLOBECOM, vol. 6, Nov. 29-Dec. 3 2004, pp. 3999-4003.

[6] Broadband Wireless Access: IEEE MAN standard, IEEE LAN/MAN Standards Committee IEEE 802.16-2004, 2004.

[7] D. Young and N. Beaulieu, "The generation of correlated rayleigh random variates by inverse discrete fourier transform," IEEE Trans. Commun., vol. 48, no. 7, pp. 1114-1127, Jul. 2000. 

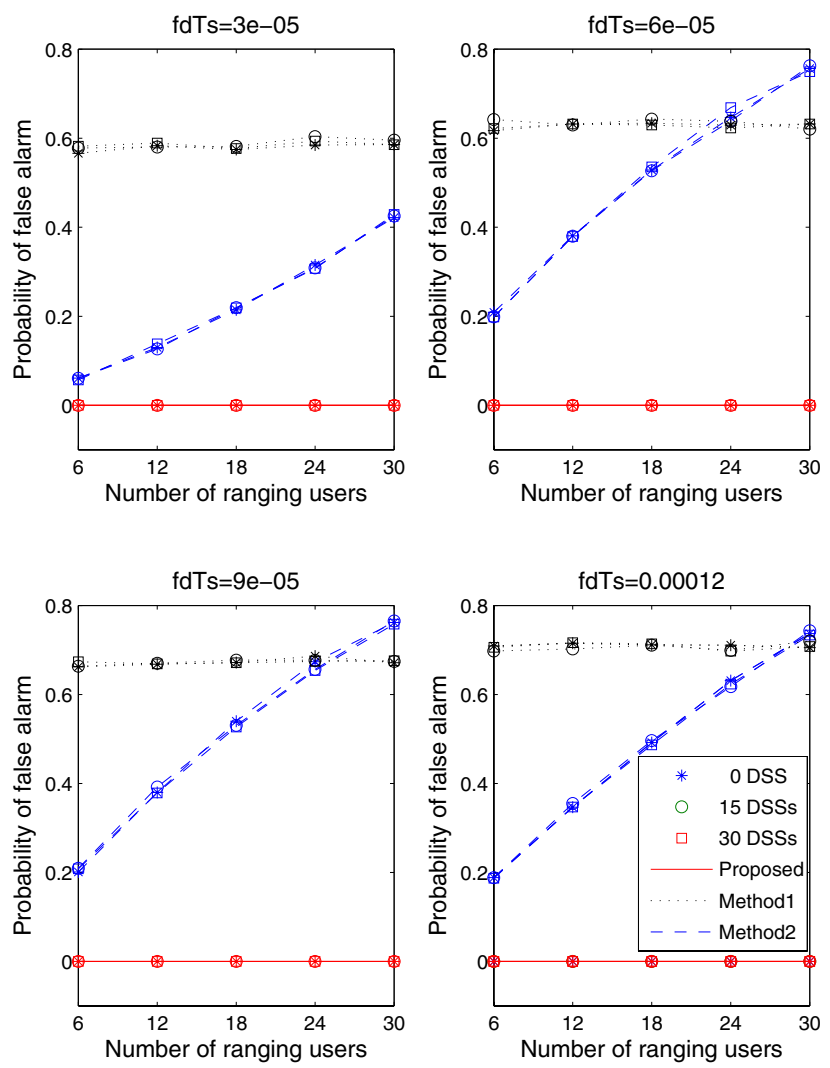

Fig. 2. The probabilities of false alarm
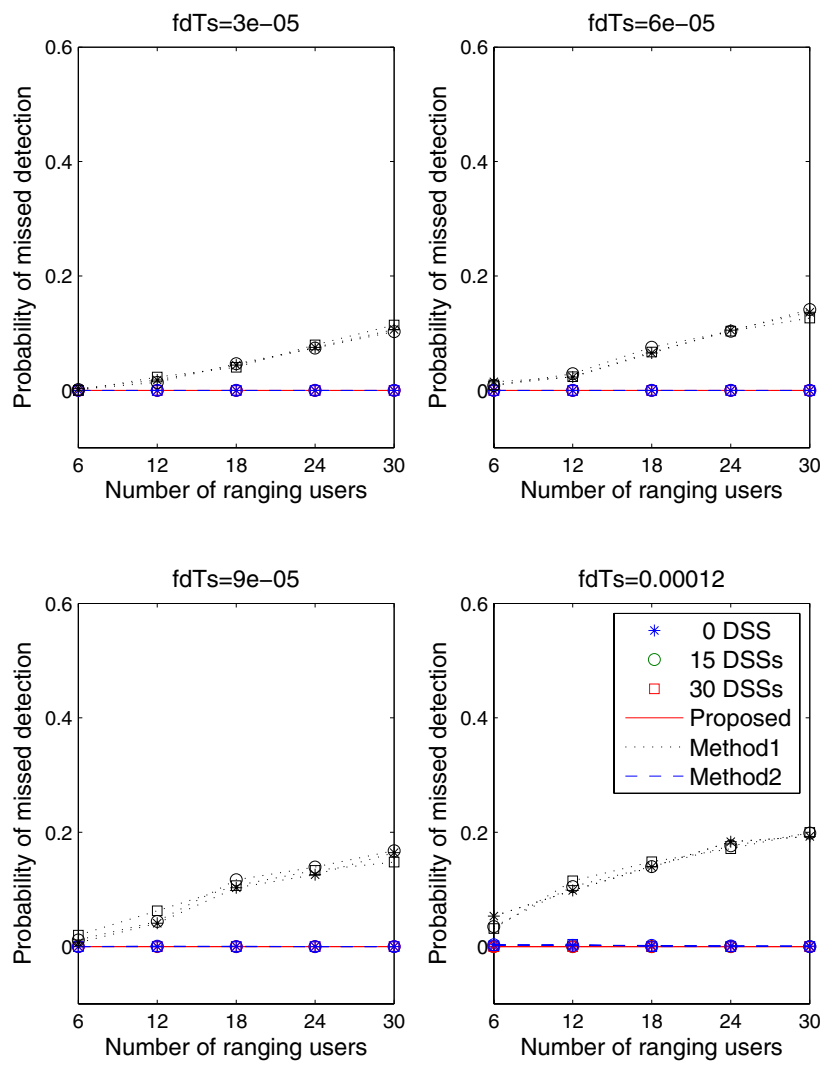

Fig. 3. The probabilities of missed detection
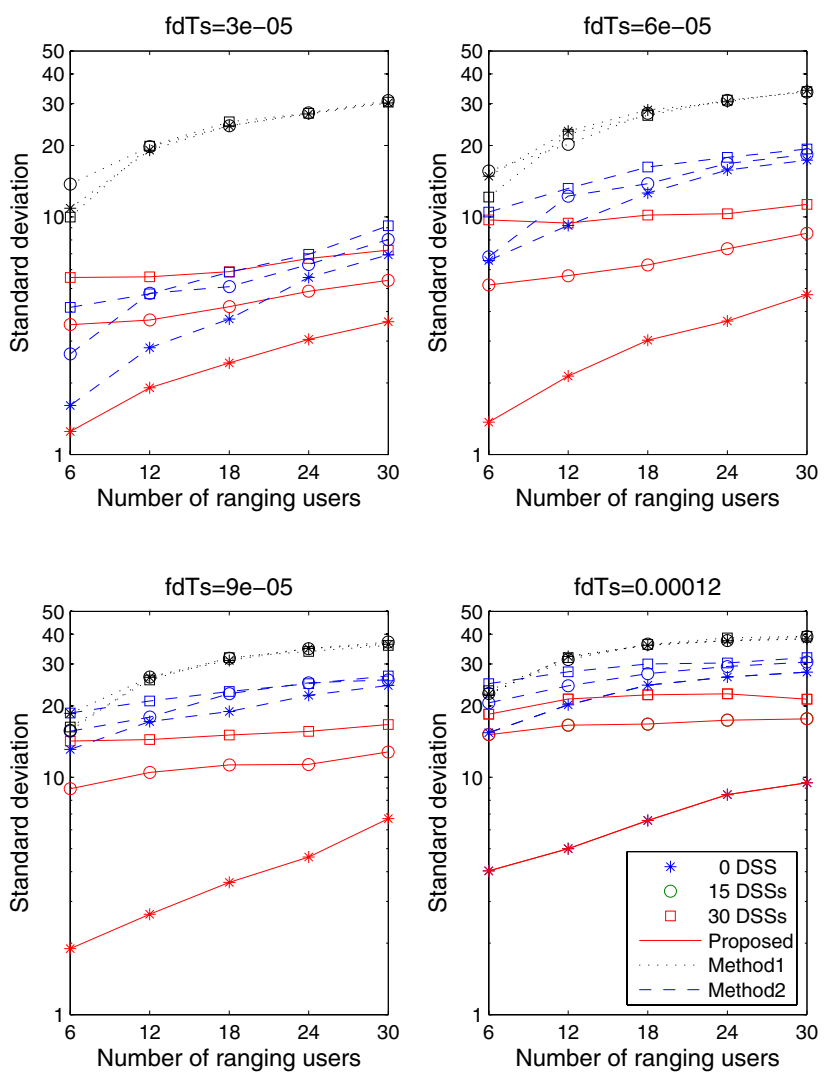

Fig. 4. Timing offset estimation performance comparison
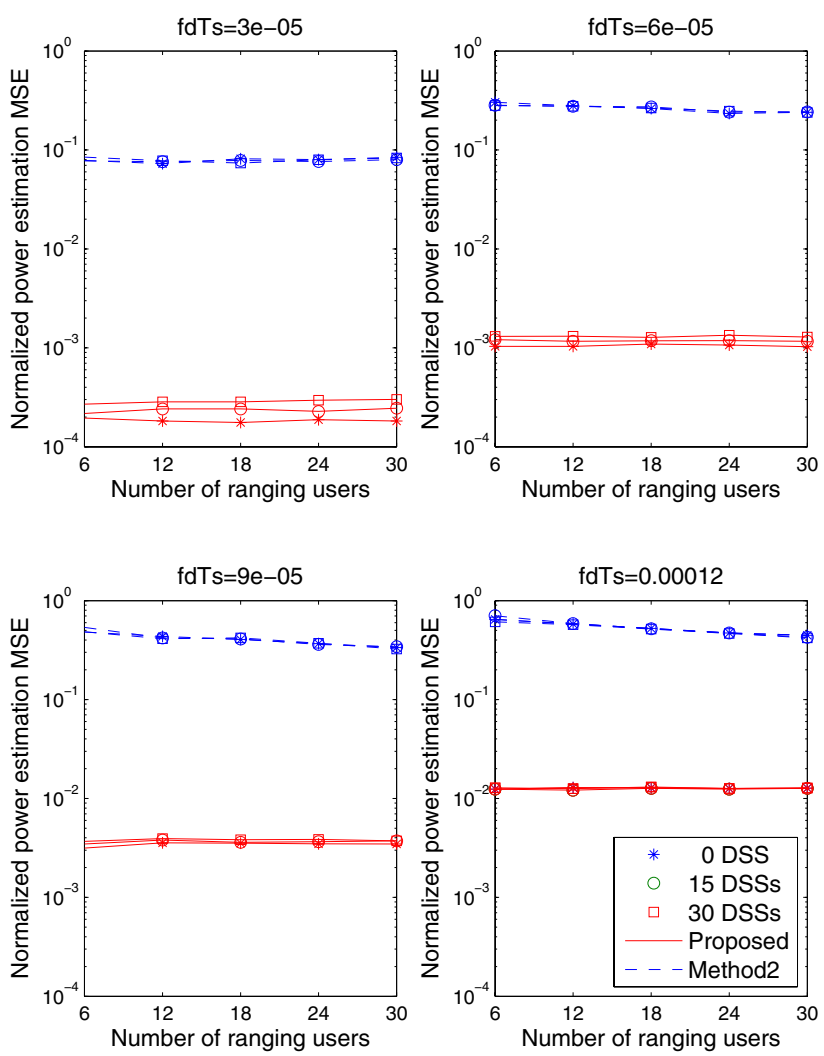

Fig. 5. Power estimation performance 